\title{
Investor Sentiment on the Stock Market using Artificial Neural Networks
}

Oana Mădălina POPESCU ${ }^{1}$

Abstract
The present study uses volatility as a measure of investor sentiment on the
Romanian capital market. The GARCH(1,1) model and the GARCH $(1,1)$ model with
Student-t innovations are used in order to describe the volatility of the Bucharest
Exchange Trading index. The estimated volatility series are afterwards included into
two artificial neural networks with the purpose to evaluate the forecasting performance
of these networks. The results show that even though the artificial neural networks are
well specified the volatility of the BET index, as measured by the GARCH(1,1) and
GARCH-t(1,1) models, does not represent a proper measure for investor sentiment on
the market.

Keywords: investor sentiment, volatility, artificial neural network, $\operatorname{GARCH}(1,1)$ model, GARCH-t $(1,1)$ model

JEL classification: $\mathrm{C} 01, \mathrm{C} 12, \mathrm{C} 13, \mathrm{G} 17, \mathrm{G} 41$

DOI: $10.24818 /$ RMCI.2019.5.508

\section{Introduction}

The way that people expose their conceptions and opinions has changed dramatically once the internet has become part of our lives, so now we can post product reviews, we can express opinions on-line, we can become members of different groups online, we can administrate blogs, all in all producing what is now called "user-generated content".

The behavior of on-line transmission represents a new quantifiable information source with many practical applications. When a person wants to buy a product, first it researches it online and evaluates the customer opinions related to the product. There is no need any more for companies to make surveys or to hire external consultants in order to find customer opinions regarding the goods produced due to the fact that user-generated content already does this.

However, finding and monitoring materials that contain opinions can be a difficult task, due to the enormous number of different sources, each source having a large volume of text that contains opinions. Sentiment analysis (opinion mining) has developed in order to satisfy this necessity. The data analyzed are obtained by "extracting" from texts sentiments (degrees of satisfaction) and key information. It

1 The Bucharest University of Economic Studies, E-mail: oana.popescu@csie.ase.ro, Telephone: 0724222293 
is said that sentiments represent the capacity of a human being to feel, discover and appreciate.

The analysis of investor sentiments on stock markets has become very popular among researchers. The advocates of passive portfolio management are sustainers of the efficient market theory, while active portfolio managers, strategic managers, analyze the movements on the market from a behavioral point of view, considering the market inefficient. More than half of the last century, the financial theory has been dominated by the efficient market hypothesis, even though many studies conclude that stock price movements are unpredictable.

Behavioral finance theoreticians use as inspiration the psychology domain, trying to find inconsistent patterns with market efficiency, or applying notions from psychology that regard decisions from the individual's point of view. Efficient market theory starts from the assumption that investors are rational and the market contains perfect information. Nowadays there is a new wave of literature about the psychological and behavioral factors that can influence stock price movements. Due to the recent financial and economic crisis, the rationality hypothesis does not hold any more, behavioral factors being used more and more in order to describe stock price variations. This is why artificial neural networks represent one of the methods used in the last years in order to simulate future movements of stock prices. In comparison to linear econometric methods, artificial neural networks can identify subtle functional relations between the variables, even though these relations are more difficult to interpret for artificial neural networks.

This is why the present study aims to identify patterns in stock price movements with the help of sentiment analysis and artificial neural networks, having as a measure of the investor sentiments the volatility on the stock market.

The rest of the paper is organized as follows: chapter 2 synthetizes the literature regarding investor sentiment analysis and artificial neural networks, whereas, chapter 3 describes the empirical findings of the study, related to investor sentiment analysis by estimating the volatility of the Bucharest Stock Exchange benchmark index (BET), as a measure of investor sentiment on the market. The volatility was estimated using the $\operatorname{GARCH}(1,1)$ model and $\operatorname{GARCH}(1,1)$ model with Student- $t$ innovations, in order to allow fatter tails for the conditional distribution. The final part of the paper presents the conclusions.

\section{Behavioral finance and investor sentiment analysis}

Behavioral finances embody psychological components in order to contradict the intensively used hypothesis of rationality from conventional finances.

The main idea behind this approach is that human nature includes both rationality and "animal spirits", the latter influencing more the behavior of investors. Keynes was the first that underlined in his book the fact that emotions can influence more human behavior than rational processes, introducing the

Volume 20, Issue 5, December 2019

Review of International Comparative Management 509 
expression "animal spirits" in order to describe economic reality as "a spontaneous urge to action rather than inaction" (Dhaoui and Khraief, 2014).

Behavioral finance researchers have tried to improve the initial model coming to a model based on two main hypotheses: (i) investors are influenced by sentiments (DeLong, Shleifer, Lawrence and Waldman, 1990) and (ii) betting against sentimental investors is risky and costly (Shleifer and Vishny, 1997). Therefore, in the language of behavioral finance there are limits to arbitrage. (Baker and Wurgler, 2007). Thus, behavioral finances propose that financial phenomena be described with the help of models that are not based on the rationality hypothesis. Formally, the effects of investor sentiments on stock returns can be explained with the help of regression models such as (Schmeling, 2008):

$$
R_{i, t+1}=\alpha_{i, t+1}+\beta_{i, t+1} \times \text { Sentiment } t_{t}+e_{i, t+1}
$$

where $R_{i, t+1}$ is the return of stock $i$ at time $t+1$ or the market return at time $t+1$, and Sentiment $t_{t}$ represents the measure used to evaluate investor sentiments at time $t$. In the literature several measures have been used in order to express investor sentiments, such as (Baker and Wurgler, 2007):

- surveys;

- investor state of spirit;

- mutual funds capital flows;

- liquidity (trading volume);

- public offerings volume;

- the return in the first day of a public offering;

- volatility.

Investor sentiments can be considered to be representative with respect to a certain norm. So, for an optimist investor ("bullish") or a pessimist one ("bearish") it is expected the return to be over/under the average return (Brown and Cliff, 2004).

"Tetlock (2007) found that the news from mass-media can predict the movements of market global indicators. He used principal component analysis in order to develop a measure of the pessimism degree in mass-media by using the content of the Wall Street Journal rubric „Abreast of the Market”, using afterwards a vector autoregressive model (VAR) in order to estimate the connection between the pessimism degree and the stock market. He concluded that high pessimism levels in mass-media predict a decreasing pressure on prices, large trading volumes and low returns."

\subsection{Investor sentiment and artificial neural networks}

Stock market price forecasting has always been an extremely difficult task due to the fact that that the dynamic of these markets is complex and nonlinear. Besides several economic factors that can affect stock markets, investor sentiments represent another factor that could influence stock market prices. Therefore,

$510 \quad$ Volume 20, Issue 5, December 2019 Review of International Comparative Management 
according to behavioral finance theory, investors make decisions with respect to their sentiments (emotions), instead of being guided by a completely rational process. Thus, stock prices can be influenced by sentiments, or otherwise said by an irrational behavior.

"Khashei and Bijari (2010) use a hybrid model with the purpose to reduce the risk associated to using an inadequate model by combining several models. Starting from autoregressive integrated moving average models (ARIMA), they estimate the necessary parameters in phase one, and in phase two they use an artificial neural network in order to model the results of the ARIMA model with the purpose to predict future values of the time series." "Lahmiri (2011) uses investor sentiments in order to predict the daily stock trend from the American technological sector using out-of sample data (not in-sample data like other studies use in order to forecast stock market prices) with the help of artificial neural networks and investor sentiment. He evaluates the performances of a probabilistic artificial neural network in comparison to the performances obtained by a backpropagation neural network in order to predict technological stock prices."

Artificial neural networks have been developed in order to replicate biological neural systems - especially the human brain and they are constituted by simple interconnected elements called neurons or nodes. Each node receives an input signal that embodies all the information received from the other nodes and from external stimulus, information that will be afterwards locally processed with the help of an activation transfer function, being thus produced an output signal to the other nodes. Artificial neural networks gather, store and recognize certain patterns using the experience gathered previously and also have the capability to evolve at the same pace as the surrounding environment. Researchers consider that these qualities give artificial networks the capability to identify patterns in stock market prices that can be used in the future to predict prices.

In the past two decades numerous models of artificial neural networks have been proposed. Among these models there are multi-layer perceptron networks (MLP), the networks developed by Hopfield (1982) and the Kohonen networks (1982). Hopfield proposed a recurrent artificial network that works the same as an associative memory (this type of memory can remember a partially distorted version of an event). These networks don't have layers and there is a complete connectivity between the nodes, the outputs of the network being stable states of an iterative process. The networks developed by Kohonen are motivated by the capacity of the human brain to self-organize.

The MLP networks are used to solve numerous problems, but mostly they are used to solve forecasting problems (Zhang, Patuwo and Hu, 1998). These networks are composed by several layers, with the following structure:

○ the first layer, or the lowest layer, is an input layer, that holds the information gathered externally;

$\circ \quad$ the last layer, or the top layer, is the output layer where the solution of the problem is obtained;

$\circ$ hidden layer that separates the input layer from the output layer. 
The nodes between the adjacent layers are completely connected in most cases through several acyclic arcs, starting from the lower layer to the superior ones.

In the case of an explicative forecasting problem, the inputs in an artificial neural network are usually independent variables. Therefore the neural network is equivalent to a regression model that is nonlinear.

Very important for an artificial neural network is to determine the arc weights, called learning process (the information gathered by the network is stored in arcs and nodes as the weights of the arcs and variations of the nodes). This process takes the form of input vectors or study patterns, each element in the input vector having one node from the input layer of the network (the number of input nodes is equal to the dimension of the input vector).

All the data included in the analysis is usually divided into two categories: the learning set (in-sample data) that is used to estimate arc weights and the test set (out-of-sample data) that is used to measure the generalization capacity of the market.

In the first phase of the learning process, the study patterns from the learning set are included in the input nodes. The activation values of the input nodes are weighted and accumulated in each node from the first hidden layer. The sum is afterwards transformed by an activation function in the activation value of the node which becomes an input into the next layer, this process continuing until the output values of activation are found. The algorithm has the objective to improve performances and minimize system errors.

\subsubsection{The training algorithm}

The training algorithm of an artificial neural network represents a nonlinear optimization problem without constraints, in which the network weights are modified iteratively in order to optimize the total mean error or the total squared error, between the desired values and the real ones of all the output nodes in comparison the input nodes. The most popular training method is the backpropagation algorithm. In the case of this algorithm, a training rate must be specified, this rate being essential in order to determine the magnitude of the changes in the weights. In comparison to this method, that has problems of inefficiency and lack of robustness (Zhang, Patuwo and Hu, 1998), the backpropagation with momentum, is the method mostly preferred by researchers.

\subsubsection{Data normalization}

Nonlinear activation functions such as the logistic function have the role to restrict the output of a node to a value comprised between $(0,1)$ or $(-1,1)$. This is usually done before the beginning of the training process. 


\subsubsection{Training sample and test sample}

The training sample is used to develop artificial neural networks and the test sample is adopted to evaluate the forecasting capacity of the model. The problem in this case is to select the data separation method between the learning set and the test set. Generally, in order to solve this problem the researcher evaluates certain factors such as the characteristics of the problem, data type, data dimension. It is very important that both sets be representative for the population they were extracted from. An improper selection of these sets will affect the optimal structure of the neural network and its performances. Most researchers select these sets following the rules: $90 \%$ vs. $10 \%, 80 \%$ vs. $20 \%, 70 \%$ vs. $30 \%$ and so on (Zhang, Patuwo and $\mathrm{Hu}, 1998)$. However, there is no consensus regarding sample dimension, the quantity of information used by the network in the learning process, the learning method, the complexity of the problem or the noise in the data. Generally, the sample volume is directly related to the desired accuracy level of the problem. A larger sample volume will allow the neural network to model any complex data structure.

\subsubsection{Measures used to evaluate the performance of the artificial neural network}

The most used methods to measure the performance of an artificial neural network are the ones that evaluate the forecasting error, that is defines as the difference between the actual (desired) value and the forecasted one (Zhang, Patuwo and $\mathrm{Hu}, 1998)$ :

- mean absolute error: $M A E=\frac{\sum\left|e_{L, E}\right|}{N}$;

- $\quad$ sum of squared error: SSE $=\sum\left(e_{\mathrm{i}, \mathrm{t}}\right)^{\mathrm{z}}$;

- mean squared error: $M S E=\frac{\sum\left(\varepsilon_{L \in E}\right)^{2}}{W}$;

- root mean squared error: $R M S E=\sqrt{M S E}$;

- mean absolute percentage error): $M A P E=\frac{1}{N} \sum\left|\frac{\mid e_{L E R}}{Y_{L E R}}\right|(100)$.

where $e_{\mathrm{i}, t}$ represents the individual forecasting error, $y_{\mathrm{i}, t}$ the actual value, and $N$ is the number of observations.

\section{Volatility as a measure to evaluate investor sentiment on the Romanian stock market}

As previously mentioned stock price forecasting represents a topic intensively researched in the financial literature. Therefore, in the following part of the paper two artificial neural networks are used in order to evaluate investor sentiment on the Romanian capital market. Volatility was chosen as a measure for investor sentiment, being estimated by using a $\operatorname{GARCH}(1,1)$ model and a GARCH$t(1,1)$ model for the daily series of returns of the BET index (the Bucharest Stock 
Exchange benchmark index). 2044 daily observations were chosen for BET in order to teach the artificial neural network for each model.

The GARCH model was independently developed by Bollerslev (1986) and Taylor (1986). This model allows the conditional variance to depend on its own error terms, such that the simplest form of the conditional variance equation is:

$$
\begin{aligned}
& R_{\mathrm{i}, t}=\sigma_{i, t} e_{i, t}, e_{i, t} \sim N\left(0, \sigma_{i, t}^{2}\right) \\
& \sigma_{i, t}^{2}=\alpha_{0}+\alpha_{1} R_{i, t-1}^{2}+\beta_{1} \sigma_{i, t-1}^{2}
\end{aligned}
$$

This is a $\operatorname{GARCH}(1,1)$ model, and the conditional variance can be interpreted as a weighted function of a long term mean value (dependent on $\alpha_{0}$ ), of the information related to the volatility of the previous period $\left(\alpha_{1} R_{i, t-1}^{2}\right)$ and of the variance from the previous period $\left(\beta_{1} \sigma_{i, t-1}^{2}\right)$. A GARCH $(1,1)$ model is sufficient to express volatility (Gujarati, 2004).

GARCH models rely on the hypothesis that the error terms follow a normal distribution, but in the case of stock market returns, the error distribution is generally characterized by fatter tails or excess kurtosis. Therefore a $\operatorname{GARCH}(1,1)$ model with Student- $t$ innovations was also estimated in order to allow excess kurtosis in the conditional distribution, the error terms for this model following a Student- $t$ distribution $\left(e_{\mathrm{i}, \mathrm{t}} \sim T_{\mathrm{v}}\left(0, \sigma_{\mathrm{i}, \mathrm{t}}^{2}\right)\right)$, instead of a normal distribution $e_{i, t} \sim N\left(0, \sigma_{i, t}^{2}\right) . T_{v}\left(0, \sigma_{i, t}^{2}\right)$ represents a Student- $t$ distribution with 0 mean, $\sigma_{i, t}^{2}$ variance and $v$ degrees of freedom. The $v$ parameter determines among others, the kurtosis degree of the conditional distribution.

The models GARCH$(1,1)$ and GARCH-t $(1,1)$ were estimated using also an $\mathrm{AR}(1)$ term in order to eliminate the serial correlation of the error terms. The results are presented in the following table:

Table 1. Models GARCH(1,1) and GARCH-t $(1,1)$

\begin{tabular}{llllrr}
\hline Model & $\alpha_{0}$ & $\alpha_{1}$ & $\beta_{1}$ & \multicolumn{1}{c}{ AR(1) } & ARCH \\
\hline GARCH & $0.0000^{8}$ & $0.1238^{8}$ & $0.8654^{8}$ & $0.0760^{8}$ & 0.6252 \\
GARCH-t & $0.0000^{8}$ & $0.1814^{8}$ & $0.8110^{*}$ & $0.0627^{88}$ & 0.5776 \\
\hline
\end{tabular}

* Significant for a $1 \%$ level of significance.

** Significant for a $4 \%$ level of significance.

*** Significant for a $10 \%$ level of significance.

Note: In the above table are summarized the results of the models $\operatorname{GARCH}(1,1)$ and GARCH-t $(1,1)$, and the ARCH Test is the LM ARCH test for conditional heteroscedasticity of order 12 .

The AR(1) term is significant in both cases. This result contradicts the weak form of market efficiency. As expected for a daily $\operatorname{GARCH}(1,1)$ model the sum of the coefficients is almost 1 . The conditional variance coefficient is almost 
0.9 , which means that the shocks of the conditional variance are persistent and that small changes in the conditional variance are followed by other small changes, large changes being followed by other large changes. The ARCH test shows no ARCH effects. The results obtained estimating the GARCH-t $(1,1)$ model are similar with the ones of the GARCH(1,1) model. Using the estimated volatility, a series of conditional volatility is generated. Volatility can be usually measured by using either variance or standard deviation. In the case of the present study was chosen the volatility summarized by variance, considering the variance of the first observation equal to the variance of the daily BET index returns series, and the squared return of the first observation equal to the expected return of the daily BET index squared returns series.

Artificial neural networks with 100 neurons in the hidden layer were afterwards developed in Matlab for both volatility models. The learning algorithm used by the network was Levenberg-Marquardt (LMA). The performance of the networks was evaluated using the $M S E$ and the correlation coefficient $R$. Moreover, $70 \%$ from the data was used for the teaching algorithm (1430 observations), the rest of $30 \%$ being divided between validation (15\% -307 observations) and tests (15\% -307 observations).
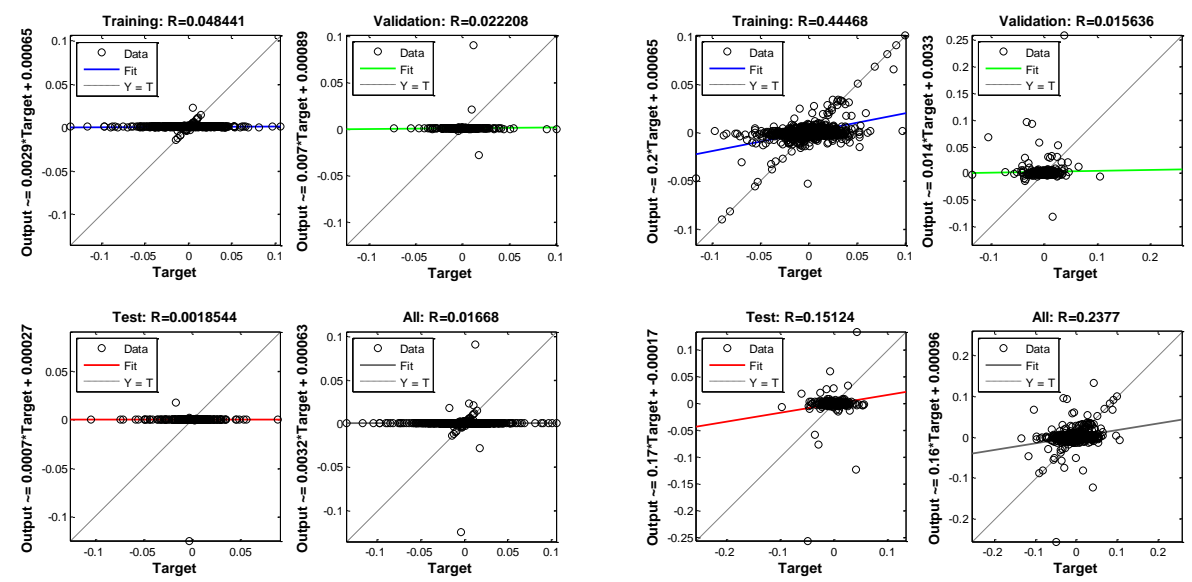

Figure 1. Regression lines for the BET index $(\operatorname{GARCH}(1,1)$, GARCH-t $(1,1))$

The $\operatorname{GARCH}(1,1)$ estimated volatility as a measure of investor sentiment at the Bucharest Stock Exchange cannot forecast the evolution of the BET index due to the fact that the correlation coefficient is almost 0 for each algorithm, so the correlation between output and targets defines a random relationship. Nor the $\operatorname{GARCH}(1,1)$ estimated volatility with Student- $t$ innovations captures the daily evolution of the BET index due to fact that the correlation coefficient for each algorithm is also very small. The values obtained for the $M S E$ show that the neural networks are well specified for both volatility models (Figure 2). 

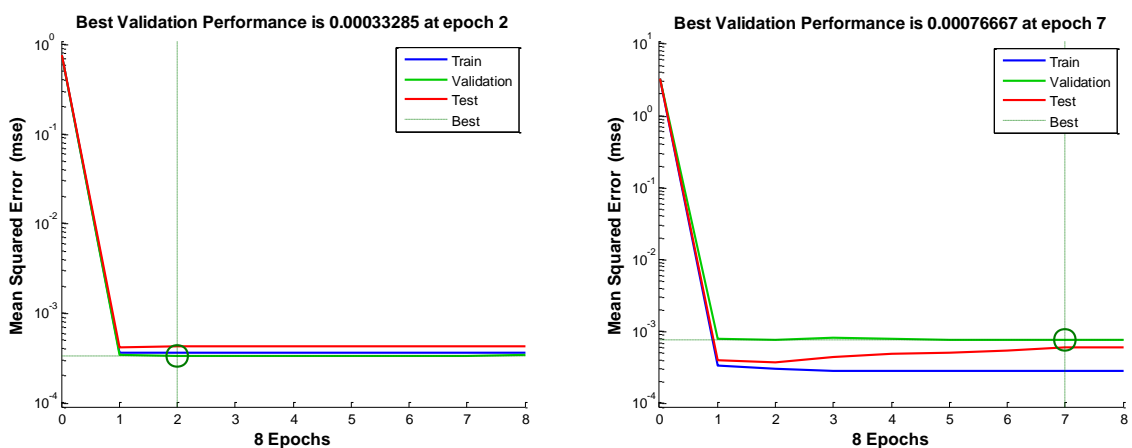

Figure 2. MSE for the BET index $(\operatorname{GARCH}(1,1), \operatorname{GARCH}-t(1,1))$

The blue line shows the MSE for the teaching set, the green one for the validation set and the red one for the test set. All three lines suggest a good performance of the network due to the fact that the error has constantly decreased while the network has continued learning. This conclusion is viable for both networks.
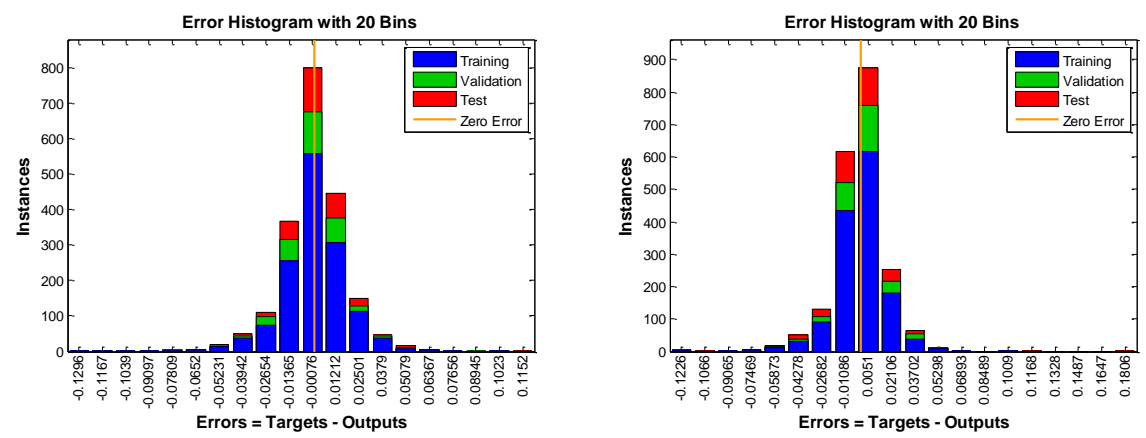

Figure 3. Error histogram for the BET index $(\operatorname{GARCH}(1,1)$, GARCH-t $(1,1))$

The histograms of the errors were also developed and they show the error distribution for the learning set, the validation set and the test set. Most errors are concentrated around 0, as shown by the orange line in Figure 3.
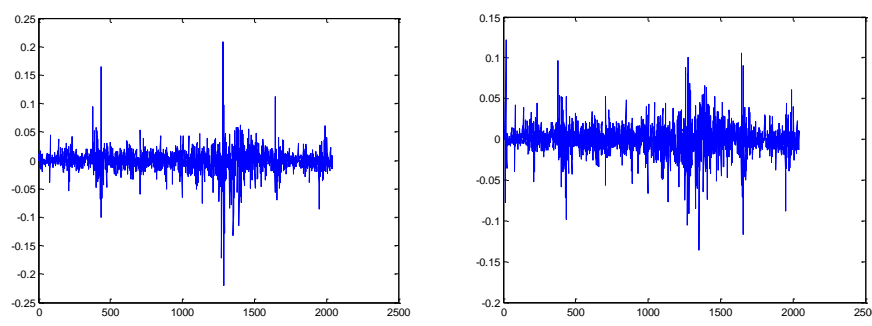

Figure 4. Error plot for the BET index 
The plot of the errors for both models (Figure 4) shows that the forecasted values by the networks vary significantly in comparison to the actual (desired) values for the BET index.

\section{Conclusions}

The efficient market theory represents the corner stone of passive portfolio management since it was initially developed by Eugene Fama (1950). In recent years, behavioral finance and sentiment analysis have become more popular among active portfolio managers that are constantly trying to identify new modalities to forecast stock market prices evolution. Moreover, artificial neural networks have become a powerful evolutionary tool for forecasting purposes. Due to the constant debate between passive and strategic portfolio managers, the present research paper estimated the volatility of the Bucharest Stock Exchange index as a measure of investment sentiment on the Romanian capital market. Two neural networks were used in the analysis, one for the $\operatorname{GARCH}(1,1)$ model and one for the $\operatorname{GARCH}(1,1)$ model with Student- $t$ innovations. Even though the results show that both networks were well specified, the results lead to the conclusion that volatility (as measured by both models) represents a poor tool for evaluating investor sentiment on the market. Also, this result does not support the hypothesis that the Romanian capital market is weakly efficient, since an AR(1) term was included in the estimation of the volatility models in order to eliminate the serial correlation. This means that the series of returns for the BET index presents short-term memory therefore a small fraction in the returns could be forecasted, this result contradicting the weak form of market efficiency. One of the major disadvantages of artificial neural networks as forecasting tools is the fact that the results are difficult to interpret, in many cases an improper selection between the learning set and the test set affecting the optimal structure of the neural network and its performances. This is why future studies regarding this topic will concentrate on different values for the teaching and test sets.

\section{References}

1. Baker, M. \& Wurgler, J. (2007). Investor Sentiment in the Stock Market, Journal of Economic Perspectives, 21, 129-151.

2. Bollerslev, T. (1986). Generalized autoregressive conditional heterosceasticity, Journal of Econometrics, 31, 307-327.

3. Brown, G.W. \& Cliff, M.T. (2004). Investor sentiment and near-term stock market. Journal of Empirical Finance, 11, 1-27.

4. Brown, S.J. \& Warner, J.B. (1980). Measuring security price performance. Journal of Financial Economics, 8, 205-258.

5. DeLong, J.B., Shleifer, A., Lawrence H.S. \& Waldman, R.J. (1990). Noise Trader Risk in Financial Markets, Journal of Political Economy, 98, 703-738.

6. Dhaoui, A. \& Khraief, N. (2014). Sensitivity of trading intensity to optimistic and pessimistic beliefs: Evidence from the French Stock Market, Arab Economics and Business Journal, 9, 115-132. 
7. Gujarati, D.N. (2004). Gujarati: Basic Econometrics, The McGraw-Hill Companies.

8. Fama, E.F. (1976). Foundations of Finance, Basic Books.

9. Hopfield, J. (1982). Neural networks and physical systems with emergetnt collective computational abilities, Proceedings of National Academy of the Sciences of the USA, 79, 2554-2558.

10. Khashei, M. \& Bijari, M. (2010). An artificial neural network (p,d,q) model for time series forecasting, Expert Systems with Applications, 37, 479-489.

11. Kohonen, T. (1982). Self-organized formation of topologically correct feature maps, Biological Cybernetics, 43, 59-69.

12. Lahmiri, S. (2011). Neural networks and investor sentiment measures for stock market trend prediction, Journal of Theoretical and Applied Information Technology, 27, 1-10.

13. Schmeling, M. (2008). Investor sentiment and stock returns: some international evidence. Discussion papers School of Economics and Management of the Hanover Leibniz University, 407, 1-42

14. Shleifer, A. \& Vishny, R. (1997). The limits of arbitrage, The Journal of Finance, 52, 35-55.

15. Taylor, S.J. (1986). Forecasting Volatility of Currency Exchange Rates, International Journal of Forecasting, 3, 159-170.

16. Tetlock, P.C. (2007). Giving Content to Investor Sentiment: The Role of Media in the Stock Market, The Journal of Finance, LXII, 1139-1168.

17. Zhang, G., Patuwo, B.E. \& Hu, M.Y. (1998). Forecasting with artificial neural networks: The state of art, International Journal of Forecasting, 14, 35-62. 